\title{
Effects of Arc Additive Manufacturing Strategies on Distribution of Residual Stress of Components
}

\author{
Bo Niu ${ }^{1, a}$, Mingxiao Shi ${ }^{2, b,{ }^{*}}$ Wengxiang Jiang ${ }^{1, c}$, YeWei Wang ${ }^{1, d}$, \\ Liqi $\mathrm{Gu}^{1, \mathrm{e}}$, and Jian Zhao ${ }^{1, \mathrm{f},{ }^{*}}$
}

\begin{abstract}
${ }^{1}$ School of Materials Engineering, Shanghai University of Engineering Science, Shanghai, China
${ }^{2}$ School of Materials Science and Engineering, Jiangsu University of Science and Technology, Jiangsu Zhenjiang, China
\end{abstract}

aemail:N916090201@163.com, bemail:smx_just@163.com, cemail:jiangwenxiang1994@163.com

*Jian Zhao, email:zhaojianhit@163.com

Keywords: Arc additive manufacturing, Strategies, Microstructures, Residual stress

\begin{abstract}
In this paper, the 304 stainless steel components were fabricated on a low carbon steel with thickness about $20 \mathrm{~mm}$ by using MIG arc additive manufacturing technology. The surface quality and the distribution characteristic of residual stress of the components prepared with three different arc additive manufacturing strategies were studied. In addition, the typical microstructures in different characteristic zones of components were revealed. The results showed that the macro features of the component with one single way strategy was highest at the initial, then it exhibited flat morphology with moderate height, and the height was lowest at the end part. However, the components with the other two strategies presented higher height at the middle parts and lower height at both ends. Specially, the maximum stress $(\sim 399.4 \mathrm{MPa})$ obtained at the onset of the component with one single way strategy. It was found that the ideal strategy with low stress could be obtained by fabricating with different strategies in adjacent layers. The typical microstructures were composed of columnar grain at the bottom, dendrite in the middle, and equiaxed grain at the top of the components.
\end{abstract}

\section{电弧增材制造路径对构件应力分布的影响 牛博 $1, a$, 石铭霄 $2, b$, 姜文祥 $1, c$, 王业伟 $1, d$, 顾立琦 $1, e$, 赵健 $1, f,{ }^{*}$ \\ 1 上海工程技术大学材料工程学院, 上海, 中国 2江苏科技大学材料科学与工程学院, 镇江, 江苏, 中国}

aemail:N916090201@163.com, bemail:smx_just@163.com, cemail:jiangwenxiang1994@163.com *赵健 邮箱:zhaojianhit@163.com

关键词：电弧增材制造; 路径; 组织; 应力

中文摘要. 文中利用MIG电弧填丝增材制造方法在 $20 \mathrm{~mm}$ 厚低碳钢板上制备 304 不锈钢熔覆 层，对三种不同增材制造路径制备的构件成形质量进行了研究，并对比研究了三种不同的增 材制造路径对构件上表层应力分布特点的影响规律, 揭示了熔覆层不同特征区典型的微观组 织。研究结果表明, 采用单一熔覆方向制备的构件成形特点表现为起始端最高, 中间稳定段 均匀平整, 高度适中, 而末端最低的特点。而其他两种路径下熔覆层成形表现为中间高, 两 端低的特点。单向熔覆获得的构件起始端应力最大为 $399.4 \mathrm{MPa}$, 采用相邻层间不同方向时获 
得的熔覆层总体应力水平较低, 是一种理想的增材制造方式。熔覆层典型组织由底部的柱状 晶, 中、上部的树枝晶与顶部的等轴晶组成。

\section{1. 引言}

增材制造技术是一种基于数字化的通过材料的填加逐层熔覆成形的整体化制造技术。与 传统制造业相比, 其实现了制造方法由 “减法” 向 “加法” 的转变, 使其在制造工艺、生产 成本与生产周期方面更具优势, 特别地该方法可以完成复杂形状构件的快速制造, 已被国家 十三五规划定位为重大发展战略 ${ }^{[1]}$ 。然而, 增材制造技术目前仍面临一些技术瓶颈尚待解决, 比如高精度制造、核心技术装备开发、缺陷控制等, 其中缓解应力集中是增材制造领域急需 突破的一个关键科学难题 ${ }^{[2]}$ 。

目前应用较多的增材制造技术主要有电弧增材制造 ${ }^{[3]}$ 、激光增材制造 ${ }^{[4]}$ 以及电子束增材制 造 ${ }^{[5]}$ 等。然而, 激光与电子束增材制造设备昂贵, 成本较高。而成本较低的电弧增材制造技 术是以电弧作为热源逐层堆砌成形金属构件, 其具有零件致密度高、冶金结合性好、化学成 分均匀、力学性能优异、能进行复杂形状制造等特点而被广泛应用于航空、航天、能源等领 域中。但电弧增材制造精度较低, 构件易发生变形, 且电弧加热区域大, 易造成堆砌过程中 热量积累严重 ${ }^{[6]}$ ，导致应力集中，因此，对电弧增材制造构件的应力进行缓释是尤为必要的。 文中采用MIG电弧作为热源通过填加304不锈钢焊丝进行增材制造，揭示不同增材制造路径下 熔覆层成形及组织特点, 主要研究不同增材制造路径对增材制造熔覆层残余应力的影响规律。

\section{2. 试验方法}

试验采用MIG填丝增材制造方法在 $20 \mathrm{~mm}$ 厚的低碳钢板上制备304不锈钢熔覆层。304不锈 钢焊丝的化学成分见表1所示, 焊丝的直径为 $\varphi 1.2 \mathrm{~mm}$. 焊前去除母材表面氧化物、油污及水分, 待干后进行增材制造, 全程氩气保护。

表1 焊丝的化学成分 (质量分数,\%)

\begin{tabular}{|c|c|c|c|c|c|c|c|}
\hline $\mathrm{C}$ & $\mathrm{Si}$ & $\mathrm{Mn}$ & $\mathrm{S}$ & $\mathrm{Ni}$ & $\mathrm{Cr}$ & $\mathrm{P}$ & $\mathrm{Fe}$ \\
\hline 0.055 & 0.60 & 1.00 & 0.005 & 8.43 & 18.20 & 0.027 & 余量 \\
\hline
\end{tabular}

试验过程中固定工艺参数不变：电压 $27 \mathrm{~V}$, 电流 $190 \mathrm{~A}$, 送丝速度 $7 \mathrm{~m} / \mathrm{min}$, 送丝角度 $90^{\circ}$, 焊接速度 $8.69 \mathrm{~mm} / \mathrm{s}$, 干伸长 $20 \mathrm{~mm}$, 电弧长度 $10 \mathrm{~mm}$, 保护气体流量 $15 \mathrm{~L} / \mathrm{min}$, 严格控制层间温 度在 $70^{\circ} \mathrm{C}$ 左右。试验中每制造一个熔覆层, 喷嘴上移一个熔覆层高度的距离再进行下一道熔 覆层的制造。选择了三种不同的增材制造路径进行对比研究增材制造路径对应力分布的影响 规律, 具体路径如图1所示。1号构件为同一增材方向熔覆10层; 2 号构件为熔覆一层后反向熔 覆第二层, 以此方法交替熔覆 10 层; 3 号构件为同一方向熔覆 5 层后反向再熔覆 5 层。对 3 种不 同构件采用小孔法进行应力测试，以TST3826静态应变测试分析系统对应变进行采集。并用 电火花切割制备试样, 采用 $\mathrm{CuSO}_{4}: \mathrm{HCl}: \mathrm{H}_{2} \mathrm{O}$ 比例为 $4 \mathrm{~g}: 20 \mathrm{ml}: 20 \mathrm{ml}$ 的溶液对熔覆层横截面 进行腐蚀, 以备进行显微组织观察。

\section{2. 试验结果及分析}

\section{1 不同增材制造路径下的熔覆层成形特点}

以不同增材制造路径制造了三个构件，每个构件由10层熔覆层组成，单层熔覆层高度约 $2 \mathrm{~mm}$ ，熔覆层整体高度 $20 \mathrm{~mm}$ ，总长度为 $210 \mathrm{~mm}$ 。具体成形质量如图1所示。

图 1（a）为路径一下的增材制造熔覆层形貌，从中可以看出构件总体成形质量良好，但 在构件起始端高度最高, 中间稳定段高度适中, 而在末端高度最低。这主要是因为在电弧电 源特性下, 熔覆末尾电弧能量逐渐下降, 熔化焊丝量相应地逐渐减少, 因此熔覆层末端的高 
度最低。而中间稳定段电弧能量能够保持稳定, 其熔化焊丝量比较固定, 所以其成形高度比 较均匀、平整。而在起始端, 一方面电弧处于起弧阶段, 电弧能量逐渐升高, 熔化的焊丝量 逐渐增多, 另一方面, 由于电弧过渡以熔滴过渡形式进入熔池, 对熔池有一定的冲击作用, 随着增材制造的进行，熔化的液态金属排向熔池的尾部，在尾部来不及回流到熔池内便快速 凝固, 因此熔覆层起始端的高度相对较高。而图 1（b）与图 1（c）在宏观形貌上比较相似, 都表现出了熔覆层中间段最高, 两端相对较低。这主要是因为, 对于图 1（b) 而言, 其增材 制造方向每一层都在交替变化, 因此相邻两层的起始端与末尾端交替对应, 因此在两端熔覆 层的高度可以得到实时的补偿, 最终形成了中间高两端低的宏观形貌, 同理, 可解释 3 号构 件成形特点。

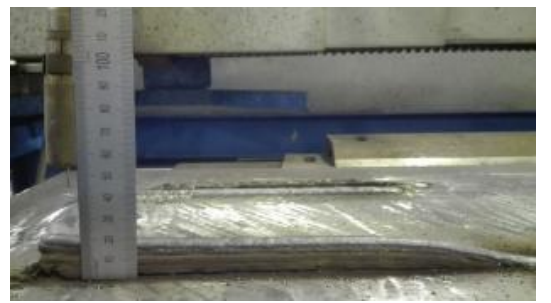

（a） 1 号构件照片

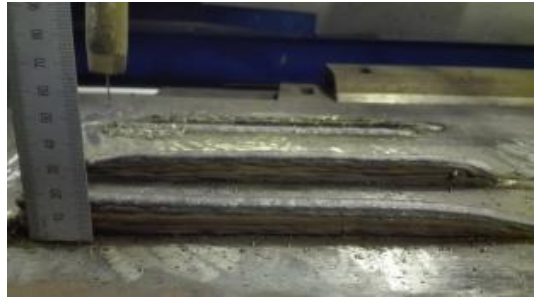

（b） 2 号构件照片

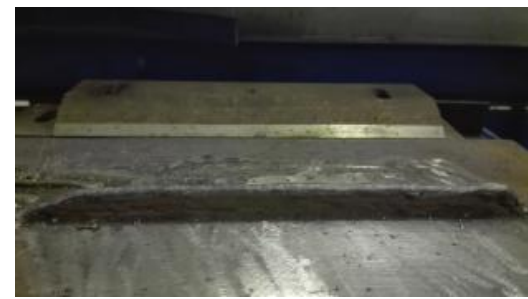

（c） 3 号构件照片

图1 三种不同增材制造路径下的构件形貌

\section{2 不同增材制造路径下的应力分布规律}

测应力前首先需要将熔覆层上表面打磨平整, 再进行应力测试, 测应力前在选好测试点 位置附近粘贴应变片, 使应变片距打孔位置 $2 \mathrm{~mm}$ 。应力测试位置如图 2 所示。其中, A 点距 离构件端部 $30 \mathrm{~mm}, \mathrm{~A}$ 点和 $\mathrm{B}$ 点距离 $65 \mathrm{~mm}, \mathrm{~B}$ 点和 $\mathrm{C}$ 点距离 $90 \mathrm{~mm}$ 。

•

A $\quad$ B $\quad$ C

第一层增材制造方向

图2 增材制造构件应力测试位置示意图

应力测试过程如图 3 所示。其中图 3（a）为应变片粘贴位置，图 3（b）为钻孔过程。对 应变仪采集的应变值，根据文献 ${ }^{[7]}$ 进行计算得到对应的应力值大小，见表 2 所示。

表2 不同增材制造路径不同位置处的应力值

\begin{tabular}{|c|c|c|c|}
\hline 位置 & $\mathrm{A}$ 点 & B 点 & C 点 \\
\hline 应力值/MPa（构件1） & 399.4 & 48.6 & 80.6 \\
\hline 应力值/MPa（构件2） & 36.7 & 131.6 & 45.8 \\
\hline 应力值/MPa（构件3） & 96.6 & 84.5 & 282.4 \\
\hline
\end{tabular}

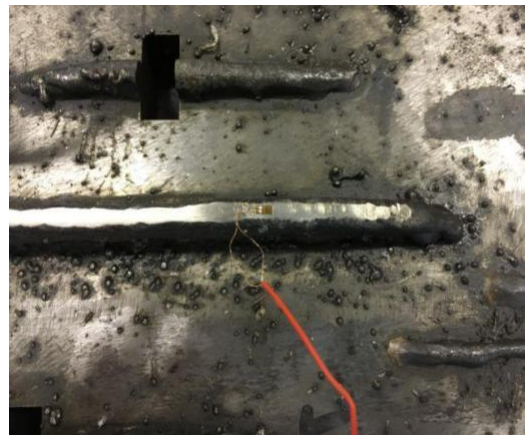

（a）应变片粘贴

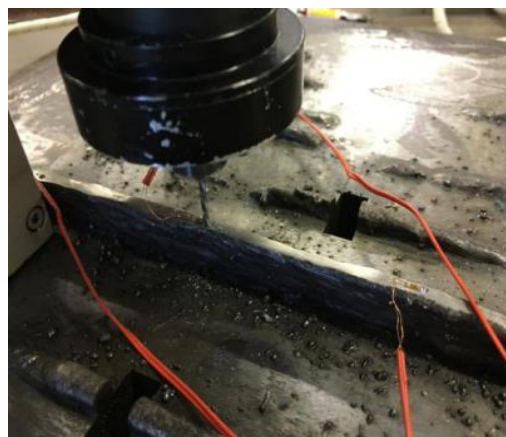

（b）钻孔过程

图3 应力测试过程照片 
图4为不同增材制造路径下的构件不同位置的纵向应力对比图。从中可以看出，1号构件 起始端即 $\mathrm{A}$ 点的纵向压应力值最大约为 $399.4 \mathrm{MPa}$ 。该处的应力值比 304 不锈钢的屈服强度更 高, 可见该处出现了塑性变形。该处应力过大的原因在于, 从宏观形貌上看该处熔覆层最高, 熔化的焊丝量最大, 在热循环反复交替过程中, 层与层之间的变形量较大, 且彼此间变形量 不均匀, 在多层熔覆过程中应力逐渐积累导致了该处应力值最大。而中间稳定段即 $\mathrm{B}$ 点处应 力值最低, 这是由于中间段能量比较稳定, 熔化焊丝量较为均匀, 层间变形量差别并不显著, 因此该处应力值约为 $48.6 \mathrm{MPa}$, 处于较低水平。而 $\mathrm{C}$ 点纵向应力值与中间稳定段水平相当, 约 为 $80.6 \mathrm{MPa}$ 。而 2 号构件纵向应力特点为中间高, 两边低, 且两侧的应力值相当。2号构件采用 了逐层交替反复熔覆的增材方式，因此，在增材制造过程当中其各熔覆层的残余应力都得到 了实时的补偿与改善, 所以其应力总体水平较低。而其中部应力较其他部位较大, 这是因为 在反复逐层的增材制造过程中其中间部位的能量较为稳定, 积累了大量的热量, 而其两端分 别处于能量的上升区和能量的下降区，由于电弧的自调节作用，其熔覆量相对较少，增材高 度上也相对较低, 所以两端积累的热量相对较少, 至使两端得残余应力较小且分布均匀。3 号构件的 $\mathrm{A}$ 点与 $\mathrm{B}$ 点的应力值处于较低水平, 且数值相差不大。而在熔覆层的 $\mathrm{C}$ 点应力值较高, 约为 $282.4 \mathrm{MPa}$ 。同样地该处的应力值也超过了 304 不锈钢的屈服强度，发生了塑性变形。3号 构件的增材制造路径是先以一个方向熔覆成形 5 层, 再以反方向连续熔覆成形 5 层。虽然该方 式熔覆层在宏观形貌上与 2 号构件相差不大, 但应力值分布却相差较大。这样的分布特点主要 是因为末端位置对应的是熔覆层后 5 层的起始端, 而我们测量的纵向应力也是熔覆层上表面的 应力值，根据 1 号试样起始端应力值特点，可以解释该处应力值应处于较高水平。

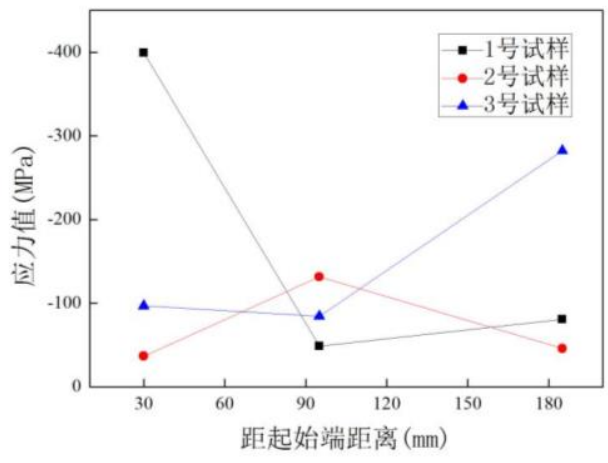

图4 不同增材制造路径下熔覆层不同位置的纵向应力

\section{3 增材制造熔覆层不同特征区组织特点}

图 5 为典型的熔覆层不同特征区的组织形貌照片, 分别为熔覆层与母材熔合线处、中下 部、中部、中上部与顶部。从图 5 (a) 可以看出, 母材与熔覆层形成了良好的冶金结合, 并 未看到组织缺陷。在大厚度母材表面, 第一层熔覆层冷却速度很快, 组织生长表现出一定的 方向性, 此时主要以柱状晶为主。从图 5 (b) 可以看出, 熔覆层中下部组织主要为树枝晶, 且这是因为虽然最大的温度梯度方向仍然为熔覆层高度方向, 但其它方向上的温度梯度比重 也有所增加所致。同时可以看出相邻两层熔覆层出现了一定的夹角, 这主要是由于由于在熔 覆过程中熔覆层的坍塌扭曲造成的最大温度梯度方向差异造成的。熔覆层中部主要由树枝晶 组成, 周围弥散分布一定量的细小 $\delta$ 铁素体。其是因为焊接时初生的 $\delta$ 铁素体与液态金属首 先发生包晶转变, 在快冷作用下从 $\gamma$ 奥氏体中析出并形成, 如图 5(c) 所示。由图 5(d) 可 以看出, 随着熔覆层高度的增加, 散热条件逐渐变差, 熔覆层中上部主要由粗大的树枝晶组 成。而在熔覆层的顶部为典型的等轴晶组织, 其形成机理为在晶体凝固的后期, 中心区域温 度均匀, 当温度降到凝固点以下时, 周围树枝晶发生断裂进入焊缝中心形核长大, 最终形成 等轴晶, 如图 5(e) 所示。 


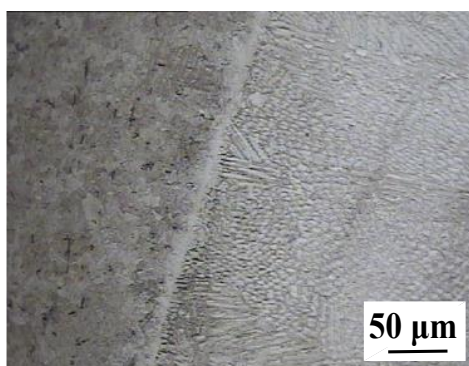

(a) 熔合线处

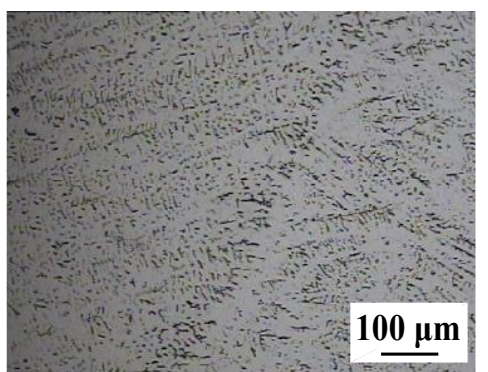

（b）熔覆层中下部

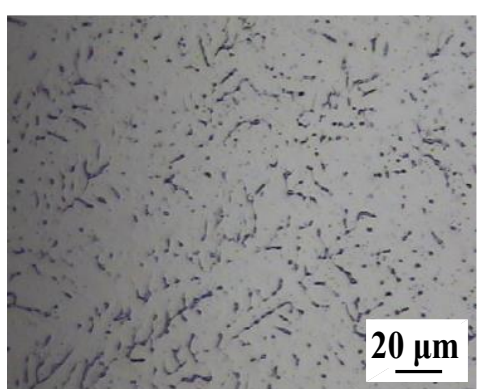

（c）熔覆层中部

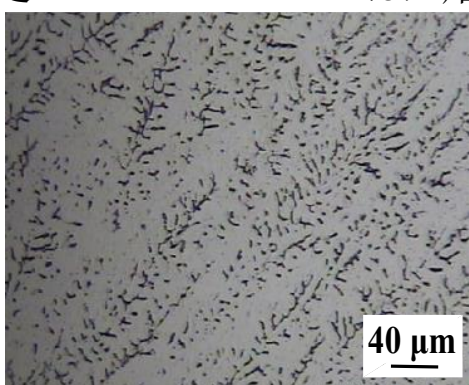

（d）熔覆层中上部

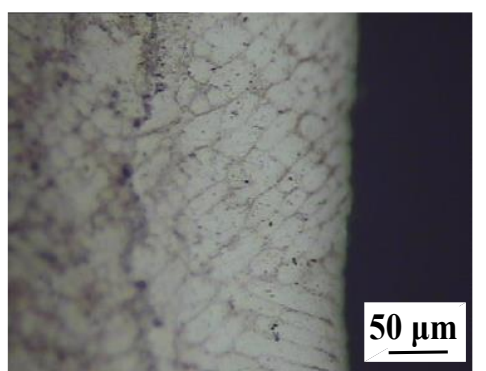

（e）熔覆层顶部

图5 熔覆层特征区的典型微观组织

\section{4. 结论}

(1) 单向熔覆时熔覆层呈现起始端最高, 中间稳定段均匀平整, 高度适中, 末端最低的 特点。而采用不同方向增材制造路径时, 得到熔覆层较为平整, 呈现中间高、两端低的特点。

(2) 单向熔覆情况下, 熔覆层起始端纵向应力最大约为 $399.4 \mathrm{MPa}$, 而采用相邻层间不同 方向时获得的熔覆层总体应力水平较低, 是一种理想的增材制造方式。

(3) 随着熔覆层高度的增加, 散热条件逐渐变差。熔覆层组织由底部的柱状晶, 中、上 部的树枝晶与顶部的等轴晶组成。

\section{References}

[1] J. Y. Lee, W. S. Tan, J. An, et al, The potential to enhance membrane module design with 3D printing technology, Journal of Membrane Science, vol.499, pp. 480-490, 2016.

[2] H. M. Wang, Materials' fundamental issues of laser additive manufacturing for high-performance large metallic components, Acta Aeronautica ET Astronautica Sinica, vol.35, pp. 2690-2698, 2014.

[3] F. C. Liu, L. H. He, X. Lin, et al, Study on microstructure and mechanical properties of arc deposition manufactured TC4 titanium alloy, Hot Working Technology, vol.43, pp. 1-5, 2014.

[4] S. Palanivel, A. K. Dutt, E. J. Faierson, et al, Spatially dependent properties in a laser additive manufactured Ti-6Al-4V component, Materials Science and Engineering: A, vol.654, pp. 39-52, 2016.

[5] C. J. Tsai, L. M. Wang, Improved mechanical properties of Ti-6Al-4V alloy by electron beam welding process plus annealing treatments and its microstructural evolution, Materials and Design, vol.60, pp. 587-598, 2014.

[6] H. B. Geng, J. T. Xiong, D. Huang, et al, Research status and trends of wire and arc additive manufacturing technology, Welding \& Joining, vol.11, pp. 17-21, 2015. 
[7] Y. Z. Guo, Experiment research on hole-drilling method for measuring residual stress, Engineering \& Test, vol.50, pp. 13-14+42, 2010. 